\title{
Intestinal carriage of extended-spectrum beta-lactamase-producing Enterobacteriaceae at admission in a Portuguese hospital
}

\author{
Marta Aires-de-Sousa ${ }^{1,2}$ • Elizeth Lopes ${ }^{2}$ - Maria Luísa Gonçalves ${ }^{3}$ - Ana Luísa Pereira ${ }^{3}$ - Augusto Machado e Costa ${ }^{4}$. \\ Hermínia de Lencastre ${ }^{2,5} \cdot$ Laurent Poirel $^{6,7,8}$
}

\begin{abstract}
To evaluate the prevalence of extended-spectrum $\beta$-lactamase (ESBL)-producing Enterobacteriaceae fecal carriers at admission in a Portuguese hospital and to determine the epidemiology and antimicrobial resistance patterns of ESBL-producing isolates. During a 2-month period, rectal swabs were collected at hospital admission from 151 at-risk patients. In addition, 48 rectal swabs were obtained from weekly screenings of 37 patients hospitalized for $>48 \mathrm{~h}$. All ESBL/carbapenemase-producing isolates were tested for antimicrobial susceptibility and characterized by PFGE and MLST. The prevalence of ESBL producers at hospital admission was $17 \%$ and $24 \%$ among at-risk patients hospitalized for $>48 \mathrm{~h}$, while the prevalence of carbapenemase producers was $3 \%$ in both cases. Most of the isolates were Escherichia coli (54\%) and Klebsiella pneumoniae (41\%). The most common ESBL identified was CTX-M-15 ( $n=17 / 34 ; 50 \%)$, followed by CTX-M-27 ( $n=10 ; 29 \%)$, CTX-M-33 $(n=4 ; 12 \%)$, SHV-12 $(n=2)$, and CTXM-55 $(n=1)$. The $20 \mathrm{E}$. coli isolates were distributed into 16 PFGE types and nine sequence types (ST), with $60 \%$ of the isolates belonging to ST131. The $15 \mathrm{~K}$. pneumoniae were grouped into 12 PFGE types and nine STs, with three STs (ST17, ST449, ST147) corresponding to $60 \%$ of the isolates. A high proportion of isolates showed resistance to ciprofloxacin (86\%), trimethoprim-sulfamethoxazole (68\%), tobramycin (57\%), and gentamicin (43\%). All isolates remained susceptible to fosfomycin. A high prevalence of ESBL-producing Enterobacteriaceae was found at hospital admission among at-risk patients and $>50 \%$ of the isolates showed resistance to first-line antibiotics for the treatment of lower urinary tract infections, leaving fosfomycin as an alternative.
\end{abstract}

Keywords ESBL $\cdot$ Enterobacteriaceae $\cdot$ Klebsiella pneumoniae $\cdot$ Escherichia coli $\cdot$ Portugal $\cdot$ Hospital admission

Marta Aires-de-Sousa

msousa@esscvp.eu

Escola Superior de Saúde da Cruz Vermelha Portuguesa (ESSCVP), Avenida de Ceuta, No 1, Edifício UrbiCeuta, 1300-906 Lisbon, Portugal

2 Laboratory of Molecular Genetics, Instituto de Tecnologia Química e Biológica António Xavier (ITQB), Universidade Nova de Lisboa (UNL), Oeiras, Portugal

3 Laboratory of Microbiology, Hospital SAMS, Lisbon, Portugal

4 Department of Medicine, Hospital SAMS, Lisbon, Portugal

5 Laboratory of Microbiology and Infectious Diseases, The Rockefeller University, New York, USA

6 Medical and Molecular Microbiology Unit, Faculty of Science and Medicine, University of Fribourg, Fribourg, Switzerland

7 INSERM European Unit (IAME, France), University of Fribourg, Fribourg, Switzerland

8 Swiss National Reference Center for Emerging Antibiotic Resistance (NARA), Fribourg, Switzerland

\section{Introduction}

The emergence of extended-spectrum $\beta$-lactamase (ESBL)-producing Gram-negative bacteria and their subsequent spread is considered a global threat. Until the 1990s, ESBL were mainly found among Klebsiella spp. and Enterobacter spp., almost exclusively in hospitals, particularly in intensive care units (ICU), and were encoded by derivatives of the $b l a_{\mathrm{TEM}}$ and $b l a_{\mathrm{SHV}}$ penicillinase genes [1]. However, in the late 1990s, the scenario basically changed with ESBL producers emerging in the community, mainly among Escherichia coli causing urinary tract infections (UTI). Meanwhile, there has been a replacement of TEM and SHV-type ESBLs by CTX-M-type enzymes $[2,3]$.

The prevalence of invasive E. coli isolates showing resistance to broad-spectrum cephalosporins is still increasing in Europe, reaching 14.9\% in 2017 [4]. Furthermore, a recent meta-analysis showed that overall, $14 \%$ of healthy individuals are colonized with ESBL-producing 
Enterobacteriaceae and $6 \%$ of these cases are in southern Europe [5].

In Portugal, the prevalence of $E$. coli invasive isolates with resistance to broad-spectrum cephalosporins was estimated at $15.6 \%$ in 2017 [4]. Several studies reported the occurrence of ESBL producers in Portugal, with a predominance of CTXM-15 not only among $K$. pneumoniae nosocomial isolates [6] but also in E. coli, both from the community and hospital settings [7]. However, there was no single prospective study evaluating ESBL-producing Enterobacteriaceae fecal carriers at hospital admission in the country, although such a study would be essential to provide a better guidance in the empiric antibiotic stewardship and infection control measures.

The aims of the present study were to prospectively evaluate the prevalence of gut carriage by ESBL-producing Enterobacteriaceae at admission in a Portuguese hospital and to determine the epidemiology and antimicrobial resistance of ESBL-producing isolates.

\section{Materials and methods}

\section{Study design}

This prospective study was conducted in a private 123-bed hospital in Lisbon, Portugal, during a 2-month period (from December 12018 to February 2 2019). During this period, all consecutive admitted patients presenting at least one risk factor for colonization/infection by ESBL producers were screened. These included patients transferred from another hospital or from a nursing home, patients with history of hospitalization or stay in a nursing home during the last 12 months, patients with previous infection with ESBLproducing Enterobacteriaceae, patients with chronic renal failure under dialysis, oncologic patients with active disease, patients with AIDS, and patients tracheostomized during the previous year, all being thereafter defined as "at-risk" patients. All such patients were rectally screened within the first $48 \mathrm{~h}$ of admission. In addition, patients at the ICU (eight beds) and Medicine Intermediate Care Unit (five beds) were screened once a week during the hospital stay. All samples were obtained from routine screenings already implemented in the hospital as infection control measures; therefore, no specific ethical concern required approval.

At hospital admission, information was obtained concerning the origin of the patient (home, nursing home, or another hospital) and previous hospitalization during the previous 12 months.

\section{Bacterial isolates}

The swabs were incubated overnight at $37^{\circ} \mathrm{C}$ in Tryptic Soy Broth (Becton, Dickinson \& Co, NJ, USA) for enrichment.
The next day, a volume of $25 \mu$ of each broth was inoculated onto two selective media: (i) CHROMagar ESBL (Frilabo, Maia, Portugal) to select for ESBL producers and (ii) ChromID Carba Smart selective medium (bioMérieux, La Balme-les-Grottes, France) to select for carbapenem-resistant isolates. The isolates were identified at the species level using the API20E system (bioMérieux).

\section{Susceptibility testing}

Antimicrobial susceptibility testing was performed using the disc diffusion method on Mueller-Hinton (MH) agar plates (Becton, Dickinson \& Co, NJ, USA) for amoxicillin, amoxicillin/clavulanic acid, temocillin, ceftazidime, cefotaxime, cefepime, cefoxitin, aztreonam, ertapenem, imipenem, amikacin, gentamicin, tobramycin, fosfomycin, trimethoprim-sulfamethoxazole (SXT), ciprofloxacin, and tigecycline, following EUCAST recommendations.

Identification of carbapenemase producers was assessed by using the Rapidec Carba NP test (bioMérieux) [8], while that of ESBL producers relied on results of the ESBL NDP test [9].

\section{Molecular analysis}

Identification of ESBL [10] and carbapenemase genes [11] was performed by PCR, as described previously, followed by sequencing of the amplicons. Standard PCR conditions were used to amplify the $q n r A, q n r B$, and $q n r S$ quinolone resistance genes [12].

The clonal relationship of the isolates was evaluated for the entire collection by pulsed-field gel electrophoresis (PFGE) as described previously [13]. Multilocus sequence typing (MLST) was performed for a representative strain of each PFGE type, and sequence types (STs) were assigned using the MLST databases for K. pneumoniae and E. coli (https:// cge.cbs.dtu.dk/services/MLST-2.0/).

\section{Conjugation experiments and plasmid analysis}

Mating-out assays were performed using the azide-resistant E. coli $\mathrm{J} 53$ as the recipient. E. coli $\mathrm{J} 53$ and $b l a_{\mathrm{KPC}-3}$ or

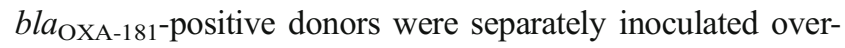
night into Luria Bertani (LB) broth $(5 \mathrm{~mL})$ and incubated. The samples were subsequently mixed at a ratio of 10:1 (donor/recipient) for $5 \mathrm{~h}$ and $100 \mu \mathrm{l}$ of this mix was deposited onto $22-\mu \mathrm{m}$ filters and incubated overnight at $37{ }^{\circ} \mathrm{C}$ onto LB agar plates. After the incubation, filters were resuspended in $\mathrm{NaCl} 0.85 \%$ and $100 \mu \mathrm{l}$ of this mixture was plated onto LB agar plates supplemented with ticarcillin $(100 \mu \mathrm{g} / \mathrm{ml})$ and azide $(100 \mu \mathrm{g} / \mathrm{ml})$. Susceptibility testing was performed for all $E$. coli transconjugants, and positivity for $b l a_{\mathrm{KPC}-3}$ or $b l a_{\mathrm{OXA}-181}$ was assessed by PCR. 
Plasmids were classified according to their incompatibility group using the PCR-based replicon typing (PBRT) method as described previously [14].

\section{Results}

During the study period, 151 patients presenting with at least one risk factor for ESBL colonization/infection were admitted to the hospital and rectal swabs from all patients were obtained within the first $48 \mathrm{~h}$. In addition, a total of 48 rectal swabs were obtained from weekly screenings of 37 patients hospitalized for more than $48 \mathrm{~h}$ ( 25 in the ICU and 12 in the Medicine Intermediate Care Unit). The rectal swabs were mainly obtained from men $(131 / 199 ; 66 \%)$ and the mean age of the patients was 74.2 years.

From a total of 199 swabs obtained, 37 ESBL-producing and/or carbapenem-resistant isolates were recovered, out of which 20 (54\%) were E. coli, 15 (41\%) were K. pneumoniae, and two were Enterobacter cloacae (Table 1).

A total of 34 isolates produced an ESBL, five isolates produced a single carbapenemase, and a single isolate coproduced two carbapenemases. The prevalence of ESBL producers at hospital admission among at-risk patients was $17 \%$ (25 isolates out of 151 admissions) and 24\% among patients hospitalized for $>48 \mathrm{~h}$ ( 9 isolates out of 37 patients), while the prevalence of carbapenemase producers was $3 \%$ in both cases (5/151 and 1/37, respectively). The most common ESBL identified was CTX-M-15 ( $n=17 / 34 ; 50 \%)$, followed by CTX-M$27(n=10 ; 29 \%)$, CTX-M-33 $(n=4 ; 12 \%)$, SHV-12 $(n=2)$, and CTX-M-55 $(n=1)$. Of note, CTX-M-27 and SHV-12 were exclusively found among $E$. coli.

Five $K$. pneumoniae isolates produced a carbapenemase, namely KPC-3 $(n=2)$ and OXA-181 $(n=3)$. In addition, a single isolate co-produced both the KPC-3 and GES-5 carbapenemases. Three isolates were co-producers of an ESBL (CTX-M-15) and a carbapenemase (KPC-3 or OXA181). Of note, the six patients colonized by a carbapenemase producer had been hospitalized during the previous year, which is known to constitute a significant risk factor.

Three patients were colonized simultaneously by different isolates (Table 1); two patients carried an ESBL-producing E. coli (CTX-M-27) in addition to an ESBL-producing $K$. pneumoniae (CTX-M-15), and a single patient carried an ESBL-producing E. coli (SHV-12), an ESBL-producing K. pneumoniae (CTX-M-15), and a carbapenemaseproducing $K$. pneumoniae (OXA-181). Two of these patients had been transferred from another hospital and the other one was living in a nursing home and had been hospitalized in this hospital during the previous year.

Two out of three patients colonized by an ESBL, or carbapenemase-producing isolate at admission who were screened one week later during their hospital stay, maintained the carriage state.

PBRT performed on respective $E$. coli transconjugants producing a carbapenemase revealed that the $b l a_{\text {OXA-181 }}$ gene was always located on an IncX3 plasmid, the $b l a_{\mathrm{KPC}-3}$ gene either on IncN and IncFII plasmid types, and the $b l a_{\mathrm{GES}-5}$ gene on a ColE1 plasmid.

Antimicrobial susceptibility testing showed that all isolates were susceptible to fosfomycin. Considering the different antibiotics tested, there was no significant difference between isolates recovered at admission or $>48 \mathrm{~h}$ of admission (Fig. 1). Most of the ESBL-producing isolates showed resistance to ciprofloxacin (86\%), SXT (68\%), tobramycin (57\%), and gentamicin (43\%).

The plasmid-mediated quinolone resistance gene $q n r S$ was identified in eight isolates, all being resistant to ciprofloxacin (including the three isolates producing OXA-181 and a single isolate producing KPC-3), while $q n r A$ and $q n r B$ genes were absent in our collection.

PFGE analysis showed a high clonal diversity, distributing the $20 \mathrm{E}$. coli, the $15 \mathrm{~K}$. pneumoniae, and the two E. cloacae isolates into 16, 12, and two PFGE types, respectively (Table 1). MLST analysis showed that the 16 E. coli PFGE types corresponded to nine STs. Interestingly, 60\% (12/20) of the $E$. coli isolates could be grouped in a single ST (ST131). The $12 \mathrm{~K}$. pneumoniae PFGE types belonged to nine STs (Table 1), out of which three (ST17, ST449, and ST147) included $60 \%$ of the isolates.

\section{Discussion}

To our knowledge, this is the first prospective study evaluating the prevalence of intestinal carriers of ESBL-producing Enterobacteriaceae at hospital admission in Portugal among at-risk patients. We report a high rate of ESBL fecal carriage at admission (17\%), highlighting the high incidence of gut colonization with ESBL producers in the community among individuals presenting risk factors for colonization/infection by multidrug-resistant bacteria. Our results highlight that the prevalence of ESBL producers in the community among atrisk patients exposed to healthcare facilities is likely high in the country in contrast to results obtained from a fecal screening conducted in 2013-2014 that revealed a very low (2\%) occurrence of ESBL producers among randomly selected healthy adults from different regions in Portugal [15]. Furthermore, the rate found in the present study (17\%) is considerably higher than the ones reported in other European countries. For instance, a recent study showed that 9\% (360/4006) of the patients admitted in a hospital in London, UK, were gut carriers of ESBL-producing Enterobacteriaceae [16]. In Madrid, Spain, the prevalence of carriers among 10,643 patients admitted to a university 


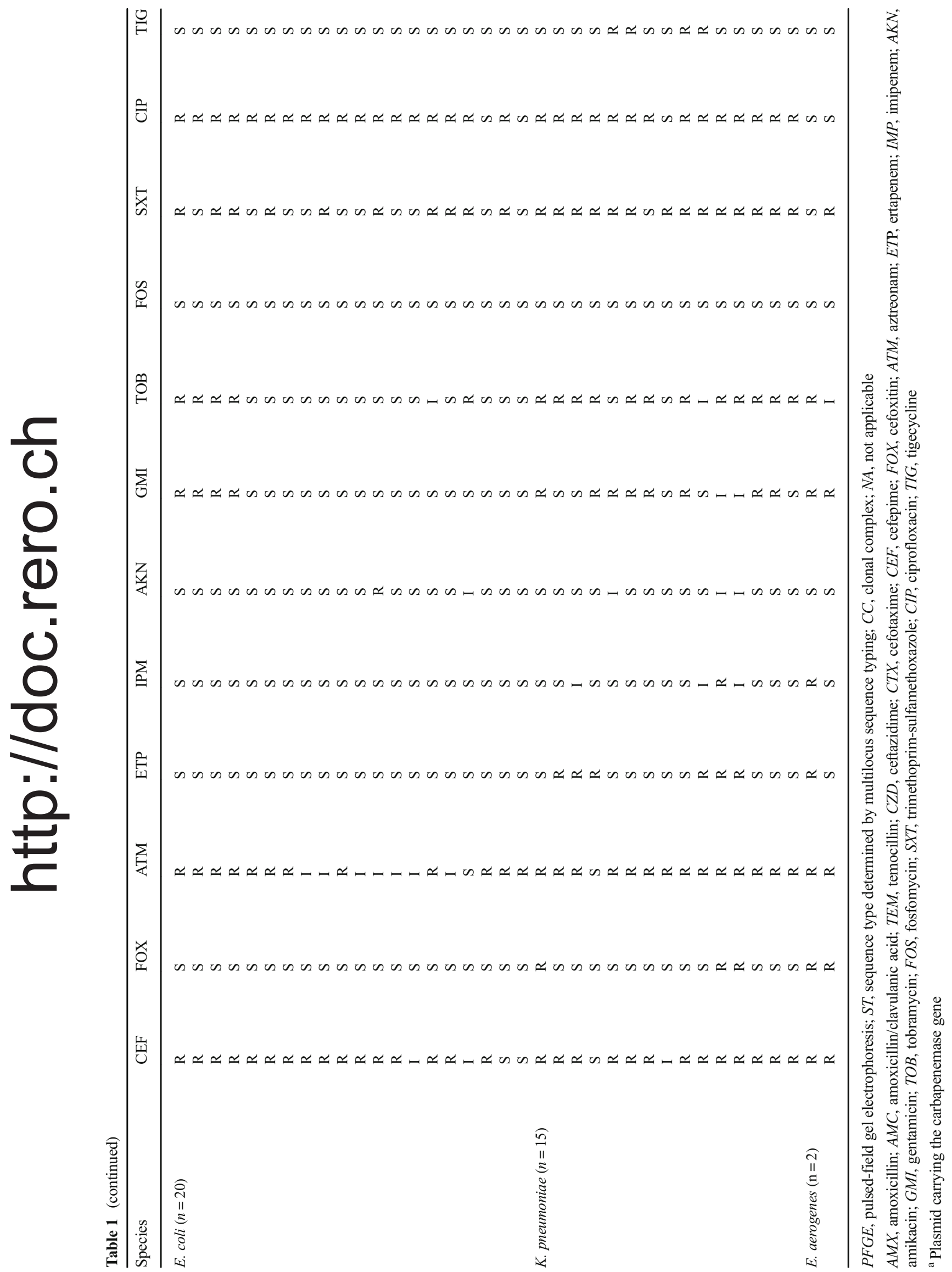




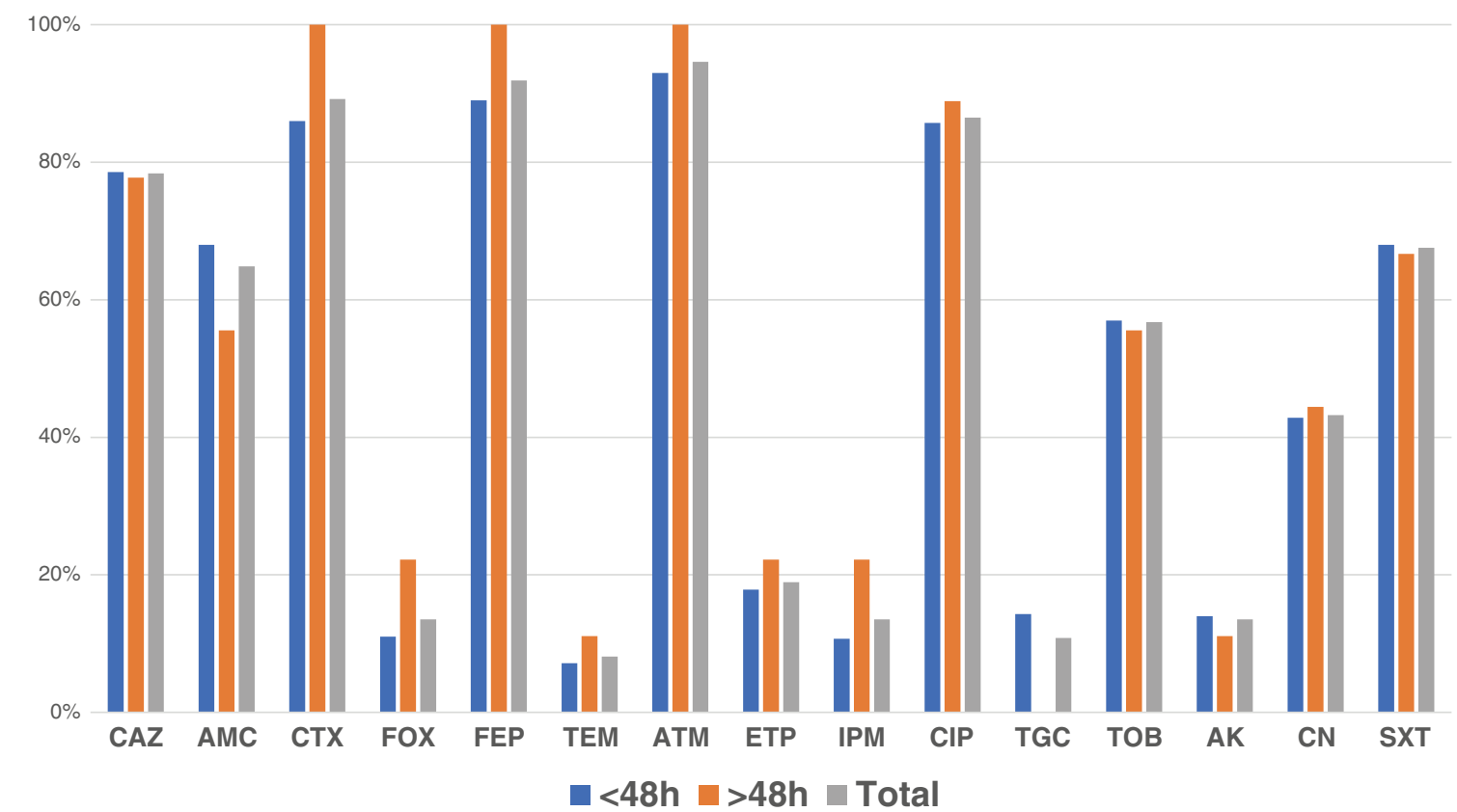

Fig. 1 Antimicrobial resistance among the 37 ESBL- and carbapenemase-producing Enterobacteriaceae isolates

hospital was $7.7 \%$ [17]. The rates were also lower in multicenter studies, such as a large admission prevalence study conducted in six German hospitals (9.5\%; 416/4376) [18] and in a study involving four hospitals in the Netherlands $(8.2 \% ; 111 / 1351)$ [19]. However, contrary to most of these studies that performed a universal screening at hospital admission, our study focused exclusively on patients presenting risk factors for colonization with ESBL producers, which might explain the higher colonization rates. Considering we investigated a private hospital, the colonization rates might be underestimated due to a low representation of lower social classes that may live in environments where carriage of ESBL producers might be higher (for instance as a consequence of poorer hygiene conditions).

E. coli was the most commonly identified ESBL producer recovered at hospital admission (54\%), while CTX-M-15 (50\%) and CTX-M-27 (29\%) were the most frequent ESBLs. Several European studies reported similar findings with $E$. coli rates varying between 68 and $89 \%$ and ESBLs of the CTX-M-1 and the CTX-M-9 groups varying between 53 and $89 \%$ and 6-21\%, respectively [16-20]. CTX-M-15 was first reported in Portugal in 2005 in a hospital in Lisbon [21] and was subsequently reported as the main ESBL among Enterobacteriaceae isolates recovered in several health care facilities from various regions $[6,22,23]$ and also in nursing homes and long-term care facilities [24]. It is actually accepted that CTX-M-15 is the most common ESBL determinant in E. coli worldwide. Noteworthy, here, we identified a CTXM-15-producing ST44 E. coli isolate, as recently identified in healthy pigs in Portugal, suggesting a possible zoonotic origin [25].
Recent surveillance studies showed that CTX-M-27 that we found in this work in almost $30 \%$ of the isolates is currently emerging in certain parts of the world, namely in Asia and Europe [26], including different hospitals and community settings in Portugal [15].

CTX-M-33, which is a CTX-M-15 derivative, has been recently shown to confer reduced susceptibility to carbapenems (mainly meropenem) and to $\beta$-lactam/ $\beta$-lactamase inhibitor combinations [27]. Interestingly, this infrequent enzyme (only reported in a single clinical E. coli in Greece in 2002 [28], in a single Aeromonas dhakensis isolate from fish in South Korea in 2014 [29] and in a K. pneumoniae isolate colonizing the gut of a patient admitted to the same Portuguese hospital in 2015 [27]), was found here among three K. pneumoniae isolates and in a single E. coli ST131 isolate, highlighting the dissemination of this ESBL gene in that hospital.

Our study showed that E. coli ST131 constitutes a major clone in the community in Portugal, harboring different ESBL encoding genes (CTX-M-15, CTX-M-27, and CTX-M-33). E. coli $\mathrm{ST} 131$ is considered the most significant high-risk clone among ESBL-producing $E$. coli since due to its predominance in many countries across the developed world, its association with multidrug resistance and virulence, and its ability to readily colonize and transmit among human hosts [30].

First-line antibiotics for the treatment of uncomplicated lower UTI include SXT, $\beta$-lactams, fluoroquinolones, nitrofurantoin, and fosfomycin [31, 32]. We found a high prevalence of ESBL-producing Enterobacteriaceae at hospital admission and more than half of the isolates showed resistance to SXT and ciprofloxacin. Therefore, the choice of empiric 
drugs to treat UTI in the community should be cautious, leaving fosfomycin as a safe alternative.

In conclusion, considering the high prevalence of ESBLproducing Enterobacteriaceae at hospital admission among patients presenting risk factors, namely recent exposition to healthcare facilities, routine screenings at hospital admission should be widely implemented in other Portuguese hospitals, enabling cohort precautions and limiting further spread of such multidrug-resistant isolates.

Acknowledgments We are grateful to Dr. Faustino Ferreira (Clinical Director) and Dra. Ana Bela Correia (Pathology Unit Coordinator) from SAMS Hospital, Lisbon, for providing us the local authorizations to perform this study.

Funding information This work was partly supported by project PTDC/DTP-EPI/0842/2014 from Fundação para a Ciência e a Tecnologia (FCT), Portugal, by Project LISBOA-01-0145-FEDER007660 (Microbiologia Molecular, Estrutural e Celular) funded by FEDER funds through COMPETE2020 - Programa Operacional Competitividade e Internacionalização (POCI), and by national funds through FCT. This work was also partially supported by ONEIDA project (LISBOA-01-0145-FEDER-016417) co-funded by FEEI - "Fundos Europeus Estruturais e de Investimento" from "Programa Operacional Regional Lisboa 2020 " and by national funds from FCT, as well as by the University of Fribourg, by the Swiss National Science Foundation (projects FNS-31003A_163432 and FNS-407240_177381). Elizeth Lopes was supported by grant 03/BI/2017 from FCT, Portugal.

\section{References}

1. Paterson DL, Bonomo RA (2005) Extended-spectrum betalactamases: a clinical update. Clin Microbiol Rev 18(4):657-686

2. Woerther PL, Burdet C, Chachaty E, Andremont A (2013) Trends in human fecal carriage of extended-spectrum beta-lactamases in the community: toward the globalization of CTX-M. Clin Microbiol Rev 26(4):744-758

3. Doi Y, Iovleva A, Bonomo RA (2017) The ecology of extendedspectrum beta-lactamases (ESBLs) in the developed world. J Travel Med 24(suppl_1):S44-S51

4. ECDC (2018) Surveillance of antimicrobial resistance in Europe annual report of the European Antimicrobial Resistance Surveillance Network (EARS-net) 2017, Stockholm, EARS-Netreport-2017-update-jan-2019pdf Cited April 10, 2019

5. Karanika S, Karantanos T, Arvanitis M, Grigoras C, Mylonakis E (2016) Fecal colonization with extended-spectrum beta-lactamaseproducing Enterobacteriaceae and risk factors among healthy individuals: a systematic review and metaanalysis. Clin Infect Dis 63(3):310-318

6. Rodrigues C, Machado E, Ramos H, Peixe L, Novais A (2014) Expansion of ESBL-producing Klebsiella pneumoniae in hospitalized patients: a successful story of international clones (ST15, ST147, ST336) and epidemic plasmids (IncR, IncFIIK). Int J Med Microbiol 304(8):1100-1108

7. Mendonca N, Leitao J, Manageiro V, Ferreira E, Canica M (2007) Spread of extended-spectrum beta-lactamase CTX-M-producing Escherichia coli clinical isolates in community and nosocomial environments in Portugal. Antimicrob Agents Chemother 51(6): 1946-1955
8. Poirel L, Nordmann P (2015) Rapidec Carba NP test for rapid detection of carbapenemase producers. J Clin Microbiol 53(9): 3003-3008

9. Nordmann P, Dortet L, Poirel L (2012) Rapid detection of extended-spectrum-beta-lactamase-producing Enterobacteriaceae. J Clin Microbiol 50(9):3016-3022

10. Ortiz de la Rosa JM, Nordmann P, Poirel L (2019) ESBLs and resistance to ceftazidime/avibactam and ceftolozane/tazobactam combinations in Escherichia coli and Pseudomonas aeruginosa. J Antimicrob Chemother In press

11. Poirel L, Walsh TR, Cuvillier V, Nordmann P (2011) Multiplex PCR for detection of acquired carbapenemase genes. Diagn Microbiol Infect Dis 70(1):119-123

12. Cattoir V, Poirel L, Rotimi V, Soussy CJ, Nordmann P (2007) Multiplex PCR for detection of plasmid-mediated quinolone resistance $q n r$ genes in ESBL-producing enterobacterial isolates. $\mathrm{J}$ Antimicrob Chemother 60(2):394-397

13. Kieffer N, Nordmann P, Aires-de-Sousa M, Poirel L (2016) High prevalence of carbapenemase-producing Enterobacteriaceae among hospitalized children in Luanda, Angola. Antimicrob Agents Chemother 60(10):6189-6192

14. Carattoli A, Bertini A, Villa L, Falbo V, Hopkins KL, Threlfall EJ (2005) Identification of plasmids by PCR-based replicon typing. J Microbiol Methods 63(3):219-228

15. Rodrigues C, Machado E, Fernandes S, Peixe L, Novais A (2016) An update on faecal carriage of ESBL-producing Enterobacteriaceae by Portuguese healthy humans: detection of the H30 subclone of B2-ST131 Escherichia coli producing CTXM-27. J Antimicrob Chemother 71(4):1120-1122

16. Otter JA, Natale A, Batra R, Tosas Auguet O, Dyakova E, Goldenberg SD, Edgeworth JD (2019) Individual- and community-level risk factors for ESBL Enterobacteriaceae colonization identified by universal admission screening in London. Clin Microbiol Infect 25(10):1259-1265

17. Diaz-Agero Perez C, Lopez-Fresnena N, Rincon Carlavilla AL, Hernandez Garcia M, Ruiz-Garbajosa P, Aranaz-Andres JM, Maechler F, Gastmeier P, Bonten MJM, Canton R (2019) Local prevalence of extended-spectrum beta-lactamase (ESBL) producing Enterobacteriaceae intestinal carriers at admission and coexpression of ESBL and OXA-48 carbapenemase in Klebsiella pneumoniae: a prevalence survey in a Spanish University Hospital. BMJ Open 9(3):e024879

18. Hamprecht A, Rohde AM, Behnke M, Feihl S, Gastmeier P, Gebhardt F, Kern WV, Knobloch JK, Mischnik A, Obermann B, Querbach C, Peter S, Schneider C, Schroder W, Schwab F, Tacconelli E, Wiese-Posselt M, Wille T, Willmann M, Seifert H, Zweigner J, Group D-AS (2016) Colonization with thirdgeneration cephalosporin-resistant Enterobacteriaceae on hospital admission: prevalence and risk factors. J Antimicrob Chemother 71(10):2957-2963

19. Platteel TN, Leverstein-van Hall MA, Cohen Stuart JW, Thijsen SF, Mascini EM, van Hees BC, Scharringa J, Fluit AC, Bonten MJ (2015) Predicting carriage with extended-spectrum betalactamase-producing bacteria at hospital admission: a crosssectional study. Clin Microbiol Infect 21(2):141-146

20. Hagel S, Makarewicz O, Hartung A, Weiss D, Stein C, Brandt C, Schumacher U, Ehricht R, Patchev V, Pletz MW (2019) ESBL colonization and acquisition in a hospital population: the molecular epidemiology and transmission of resistance genes. PLoS One 14(1):e0208505

21. Conceicao T, Brizio A, Duarte A, Lito LM, Cristino JM, Salgado MJ (2005) First description of CTX-M-15-producing Klebsiella pneumoniae in Portugal. Antimicrob Agents Chemother 49(1): $477-478$

22. Jones-Dias D, Manageiro V, Ferreira E, Louro D, ARSPiPA participants, Canica M (2014) Diversity of extended-spectrum and 
plasmid-mediated AmpC beta-lactamases in Enterobacteriaceae isolates from Portuguese health care facilities. J Microbiol 52(6): 496-503

23. Fernandes R, Amador P, Oliveira C, Prudencio C (2014) Molecular characterization of ESBL-producing Enterobacteriaceae in northern Portugal. ScientificWorldJournal 2014:782897

24. Goncalves D, Cecilio P, Ferreira H (2016) Nursing homes and longterm care facilities: reservoirs of CTX-M-15-producing Escherichia coli O25b-ST131 in Portugal. J Glob Antimicrob Resist 7:69-71

25. Fournier C, Aires-de-Sousa M, Nordmann P, Poirel L (2019) Occurrence of CTX-M-15 and MCR-1-producing Enterobacterales in pigs, Portugal; evidences of direct links with antibiotic selective pressure. Int J Antimicrob Agents In press

26. Peirano G, Pitout JDD (2019) Extended-spectrum beta-lactamaseproducing Enterobacteriaceae: update on molecular epidemiology and treatment options. Drugs 79(14):1529-1541

27. Poirel L, Ortiz de la Rosa JM, Richard A, Aires-de-Sousa M, Nordmann P (2019) CTX-M-33, a CTX-M-15 derivative conferring reduced susceptibility to carbapenems. Antimicrob Agents Chemother In press
28. Galani I, Souli M, Chryssouli Z, Giamarellou H (2007) Detection of CTX-M-15 and CTX-M-33, a novel variant of CTX-M-15, in clinical Escherichia coli isolates in Greece. Int J Antimicrob Agents 29(5):598-600

29. Yi SW, Chung TH, Joh SJ, Park C, Park BY, Shin GW (2014) High prevalence of blaCTX-M group genes in Aeromonas dhakensis isolated from aquaculture fish species in South Korea. J Vet Med Sci 76(12):1589-1593

30. Pitout JD, DeVinney R (2017) Escherichia coli ST131: a multidrug-resistant clone primed for global domination. F1000Res 6

31. Jancel T, Dudas V (2002) Management of uncomplicated urinary tract infections. West J Med 176(1):51-55

32. Kang CI, Kim J, Park DW, Kim BN, Ha US, Lee SJ, Yeo JK, Min SK, Lee H, Wie SH (2018) Clinical practice guidelines for the antibiotic treatment of community-acquired urinary tract infections. Infect Chemother 50(1):67-100 